\title{
CRECIMIENTO DE ESPECIES ARBÓREAS TROPICALES EN LA COLECCIÓN DE LA UNIVERSIDAD TÉCNICA ESTATAL DE QUEVEDO
}

\author{
Pedro Suatunce Cunuhay ${ }^{1,2}$, Gorki Díaz Coronel ${ }^{1,3}$ y ${ }^{\bullet}$ Luz García Cruzatty ${ }^{1}$ \\ ${ }^{1}$ Unidad de Investigación Científica y Tecnológica, Universidad Técnica Estatal de Quevedo, $\mathrm{km} 1$ 11/2 vía \\ Quevedo-Santo Domingo de los Tsáchilas, C. P. 73. Quevedo, Los Ríos, Ecuador. ${ }^{1}$ uzcecilia29@hotmail.com \\ ${ }^{2}$ Facultad de Ciencias Ambientales, Universidad Técnica Estatal de Quevedo, km 1 11/2 vía a Santo Domingo \\ de los Tsáchilas, C. P. 73. Quevedo, Los Ríos, Ecuador. \\ ${ }^{3}$ Facultad de Ciencias Pecuarias, Universidad Técnica Estatal de Quevedo, km 7 vía a El Empalme \\ Mocache, Los Ríos, Ecuador
}

\begin{abstract}
RESUMEN
L os resultados de crecimiento de 40 especies arbóreas tropicales de la UTEQ, ubicada en la Finca "La Represa", Quevedo, Ecuador. Se establecieron en cuatro fases diferentes con 10 especies cada una. Cada especie fue considerada como un tratamiento y se empleó un diseño de bloques al azar con dos repeticiones. Cada parcela tiene una superficie de $225 \mathrm{~m}^{2}(15 \times 15 \mathrm{~m})$. Se midió el diámetro y la altura de los árboles en cada tratamiento. Se encontró diferencias significativas entre las especies para el diámetro, altura y volumen de los árboles. Además, se presenta el periodo de floración y fructificación de algunas especies.
\end{abstract}

Palabras claves: Altura, conservación, crecimiento, diámetro, fenología.

\begin{abstract}
$\mathrm{T}$ he growth performance of 40 tropical tree species UTEQ, located in "La Represa" farm near by Quevedo, Ecuador are presented. Four different phases were established with 10 species each one. Each specie were considered as a treatment and a random blocks design with two replications was used. Each plot has a surface of $225 \mathrm{~m}^{2}(15 \times 15 \mathrm{~m})$. Diameter and height of the trees was measured in each treatment. Significant differences among the species were found for the diameter, height and volume of the trees. It is also presented the flowering and fruiting periods of some species.
\end{abstract}

Key words: Height, conservation, growing, diameter, phenology.

\section{INTRODUCCIÓN}

$\mathrm{L}$ a extinción de las especies forestales es un asunto cotidiano. En épocas pasadas, este hecho se debió a causas naturales, pero actualmente las actividades humanas influyen en un alto porcentaje a la tasa de extinción (Louzada y De Souza, 1999). Los bosques tropicales son los más amenazados (Myers, 1984; Peacock, 1995). Esta situación obliga a realizar esfuerzos tendientes a buscar soluciones técnicas y prácticas (Fundación Natura, 1985).

En el Ecuador, los bosques tropicales tienen una gran diversidad de especies de flora. (Fundación Natura, 1985). Sin embargo, el estudio de los recursos genéticos forestales nativos ha sido muy limitado (Jadan y Valarezo, 1983), siendo muy escasos estudios que registren datos de crecimiento y fenología. Para facilitar el estudio de las especies forestales se hace necesario concentrarlas en un solo sito, donde se pueda preservar, investigar, tomar muestras dendrológicas, obtener información sobre la biología, fenología y los aspectos

Recibido: Junio, 2009. Aceptado: Octubre, 2009.

Publicado como ARTÍCULO en Ciencia y Tecnología 2(2): 21-27. 2009 silviculturales, y para que sirvan como fuente de semilla o material vegetativo para la propagación (INEFAN, 1994). Ante esta situación, la Unidad de Investigación Científica y Tecnológica (UICYT) de la UTEQ estableció una "Colección de especies arbóreas Tropicales" con el fin de fomentar su conservación, y evaluar su crecimiento y fenología. Hasta la presente cuenta con cincuenta especies del bosque húmedo tropical, de las cuales en el presente documento se presentan los resultados de 40 especies.

\section{MAteriales Y MÉtodos}

$\mathrm{L}$ a Colección de especies arbóreas se encuentra ubicada en la Finca Experimental "La Represa" de la Universidad Técnica Estatal de Quevedo, localizada en el recinto Fayta, parroquia San Carlos, cantón Quevedo, provincia de Los Ríos. El sitio corresponde a la zona ecológica bh-T, altitud $90 \mathrm{msnm}$, precipitación anual de $2178 \mathrm{~mm}$, temperatura media diaria de $24.5^{\circ} \mathrm{C}$, heliofanía de $7.52 \mathrm{~h} / \mathrm{d}$. La topografía es relativamente plana, con $\mathrm{pH}$ de 6.5 - 7.0; textura del suelo franco - arcilloso 
y humedad relativa de $84 \%$. El proyecto se inició en enero de 1996 estableciendo las diez primeras especies, correspondientes a la primera fase; en el año 1997 se estableció la segunda fase con diez especies. Así mis- mo, en 1998 se estableció la tercera fase y recién el año 2002 se plantaron las diez especies correspondientes a la cuarta fase (Cuadro 1).

Cuadro 1. Especies forestales de la colección de la Universidad Técnica Estatal de Quevedo

\begin{tabular}{|c|c|c|c|}
\hline PRIMERA FASE & SEGUNDA FASE & TERCERA FASE & CUARTA FASE \\
\hline $\begin{array}{l}\text { Anacardium exelsum } \\
\text { (Marañón) }\end{array}$ & $\begin{array}{l}\text { Sapindus saponaria } \\
\text { (Jaboncillo) }\end{array}$ & $\begin{array}{c}\text { Cinometra bauhiniifolia } \\
\text { (Cocobolo) }\end{array}$ & $\begin{array}{l}\text { Nectandra spp. } \\
\text { (Jigua canelo) }\end{array}$ \\
\hline $\begin{array}{l}\text { Albizia guachapele } \\
\text { (Guachapelí) }\end{array}$ & $\begin{array}{l}\text { Triplaris cumingiana } \\
\text { (Fernansánchez) }\end{array}$ & $\begin{array}{l}\text { Tabebuia guayacan } \\
\text { (Guayacán negro) }\end{array}$ & $\begin{array}{l}\text { Myroxilum balsamum } \\
\text { (Sándalo) }\end{array}$ \\
\hline $\begin{array}{c}\text { Tabebuia pentaphylla } \\
\text { (Roble) }\end{array}$ & $\begin{array}{l}\text { Cordia macrantha } \\
\text { (Laurel prieto) }\end{array}$ & $\begin{array}{l}\text { Clorophora tinctoria } \\
\text { (Moral fino) }\end{array}$ & $\begin{array}{l}\text { Zanthoxylum tachuelo } \\
\text { (Tachuelo) }\end{array}$ \\
\hline $\begin{array}{l}\text { Ocotea floribunda } \\
\text { (Cedro) }\end{array}$ & $\begin{array}{l}\text { Artocarpus altilis } \\
\text { (Fruta de pan) }\end{array}$ & $\begin{array}{l}\text { Licania arborea } \\
\text { (Guayjí) }\end{array}$ & $\begin{array}{l}\text { Carapa guianensis } \\
\text { (Tangare) }\end{array}$ \\
\hline $\begin{array}{l}\text { Cordia alliodora } \\
\text { (Laurel blanco) }\end{array}$ & $\begin{array}{l}\text { Ochroma lagopus } \\
\text { (Balsa) }\end{array}$ & $\begin{array}{l}\text { Rollinia mucosa } \\
\text { (Anona) }\end{array}$ & $\begin{array}{c}\text { Erythrina poeppigeana } \\
\text { (Bombón) }\end{array}$ \\
\hline $\begin{array}{l}\text { Colubrina arborescens } \\
\text { (Caoba de montaña) }\end{array}$ & $\begin{array}{l}\text { Cordia eriostigma } \\
\text { (Tutumbe) }\end{array}$ & $\begin{array}{l}\text { Cananga odorata } \\
\text { (Cananga) }\end{array}$ & $\begin{array}{l}\text { Simira ecuadorensis } \\
\text { (Colorado) }\end{array}$ \\
\hline $\begin{array}{l}\text { Leucaena leucocephala } \\
\text { (Leucaena) }\end{array}$ & $\begin{array}{l}\text { Cybistax donnel-smithii } \\
\text { (Guayacán blanco) }\end{array}$ & Pithecellobium sp. & $\begin{array}{c}\text { Swietenia macrophilla } \\
\text { (Caoba) }\end{array}$ \\
\hline $\begin{array}{l}\text { Gmelina arborea } \\
\text { (Melina) }\end{array}$ & $\begin{array}{c}\text { Centrolobium paraense } \\
\text { (Amarillo) }\end{array}$ & $\begin{array}{l}\text { Apeiba aspera } \\
\text { (Peine de mono) }\end{array}$ & $\begin{array}{l}\text { Gliricidia sepium } \\
\text { (Matarratón) }\end{array}$ \\
\hline $\begin{array}{l}\text { Vitex gigantea } \\
\text { (Pechiche) }\end{array}$ & $\begin{array}{l}\text { Matisia cordata } \\
\text { (Zapote) }\end{array}$ & $\begin{array}{l}\text { Cassia fistula } \\
\text { (Caña fístula) }\end{array}$ & $\begin{array}{l}\text { Talisia setigera } \\
\text { (Cebo de mico) }\end{array}$ \\
\hline $\begin{array}{l}\text { Sikingia stanleyi } \\
\text { (Manglillo) }\end{array}$ & $\begin{array}{c}\text { Inga espectabilis } \\
\text { (Guabo de machete) }\end{array}$ & $\begin{array}{l}\text { Brownea herthae } \\
\quad \text { (Clavellino) }\end{array}$ & $\begin{array}{c}\text { Erythrima smithiana } \\
\text { (Caraca) }\end{array}$ \\
\hline
\end{tabular}

A cada especie forestal se la considera como un tratamiento. Los tratamientos se encuentran establecidos en un diseño de bloques al azar con dos repeticiones. Las parcelas tienen una superficie de $225 \mathrm{~m}^{2}(15 \mathrm{x}$ $15 \mathrm{~m}$ ) y los árboles están plantados a una distancia de 3 x $3 \mathrm{~m}$. El área de la parcela útil es de $81 \mathrm{~m}^{2}(9 \times 9 \mathrm{~m})$. La parcela útil contiene nueve árboles, de los cuales se registran datos anuales de DAP (diámetro a altura del pecho) y altura total a cinco árboles, en forma de zig zag. Para la fenología se observan dos árboles en cada parcela.

Cabe destacar que para el cálculo del volumen obtenido en los cuadros anteriores y en las figuras del incremento medio anual de los volúmenes, se empleó la altura total de las especies en estudio y el factor de forma 0.629 , como valor estándar estimado por INEFAN, citado por López (2002).

\section{RESUltadOS Y DiscusióN}

Diámetros, alturas y volúmenes de las especies arbóreas tropicales de la colección de la UTEQ.

$\mathrm{E}^{\mathrm{s}}$ n la primera fase, a los 11 años de edad, la especie con mayor diámetro, altura y volumen promedio fue G. arborea Roxb. (Melina). Esta es una especie introducida que se ha adaptado fácilmente y presenta un rápido crecimiento, lo cual concuerda con (Roncacio, 2001) quien menciona a la melina como una especie de rápido crecimiento inicial. La especie con menor crecimiento volumétrico fue T. pentaphilla Bertol A. DC. (Roble), el crecimiento de ésta especie es de rápido a lento, dependiendo del sitio. En Puerto Rico, en un sistema silvopastoril de cultivos en callejones a los 14 años de edad los árboles tenían $14 \mathrm{~m}$ de altura, con $13 \mathrm{~cm}$ de dap (CATIE, 2004) (Cuadro 2).

En la segunda fase, a los 10 años de edad, la especie con mayor diámetro, altura y volumen promedio fue $O$. lagopus $\mathrm{Sw}$. (Bbalsa), siendo ésta una especie pionera que tiene un crecimiento muy rápido cuando las condiciones climáticas y de suelo son favorables (CAQ, 1992). La especie de menor crecimiento en diámetro, altura y volumen promedio fue $S$. saponaria L. (Jaboncillo), este es un árbol pequeño a mediano que alcanza hasta los $16 \mathrm{~m}$ de altura, con copa amplia que se ramifica a poca altura (CATIE, 2004) (Cuadro 3).

En la tercera fase, a los 9 años de edad, la C. odorata Lam. (Cananga) presentó el mayor crecimiento promedio en diámetro, altura y volumen; esta especie introducida y de muy rápido crecimiento, se ha adap- 
tado fácilmente a las condiciones del bosque húmedo tropical (Manner \& Elevitch, 2006). Entre las especies nativas, $A$. aspera Aubl. (Peine de mono) y $C$. tinctoria Benth. (Moral fino) sobresalieron en diámetro, altura y volumen. El moral fino en el Ecuador es una especie de aprovechamiento condicionado que por su dureza es muy cotizada y se utiliza mucho en nuestro medio para estructuras (Bachmann, 2008). La especie que presentó menor crecimiento en diámetro, altura y volumen fue C. bauhiniifolia Benth (Cocobolo), árbol o arbusto de 4 a $15 \mathrm{~m}$ de altura y de lento crecimiento (Zamora et al., 2000) (Cuadro 4).

Cuadro 2. Diámetro, altura y volumen* de especies arbóreas tropicales de la primera fase a los 11 años de edad. Quevedo, 2007.

\begin{tabular}{|c|c|c|c|c|c|c|}
\hline \multirow{2}{*}{$\frac{\text { Especies }}{\text { 1. Anacardium exelsum }}$} & \multicolumn{2}{|c|}{ Diámetro (cm) } & \multicolumn{2}{|c|}{ Altura (m) } & \multicolumn{2}{|c|}{ Volumen $\left(\mathrm{m}^{3}\right)$} \\
\hline & 17.20 & $\mathrm{bc}^{*}$ & 17.21 & bcde & 0.25 & bc \\
\hline 2. Albizia guachapele & 12.50 & $\mathrm{c}$ & 13.82 & ef & 0.11 & $\mathrm{c}$ \\
\hline 3. Tabebuia pentaphylla & 13.05 & $\mathrm{c}$ & 12.44 & $\mathrm{f}$ & 0.11 & $\mathrm{c}$ \\
\hline 4. Ocotea floribunda & 16.95 & bc & 16.89 & bcde & 0.24 & bc \\
\hline 5. Cordia alliodora & 15.65 & bc & 15.70 & cdef & 0.19 & bc \\
\hline 6. Colubrina arborescens & 16.05 & bc & 18.50 & bc & 0.24 & bc \\
\hline 7. Leucaena leucocephala & 19.80 & $\mathrm{~b}$ & 19.75 & $\mathrm{ab}$ & 0.39 & $\mathrm{~b}$ \\
\hline 8. Gmelina arborea & 31.35 & $\mathrm{a}$ & 22.60 & $\mathrm{a}$ & 1.10 & $\mathrm{a}$ \\
\hline 9. Vitex gigantea & 14.35 & bc & 14.48 & def & 0.15 & bc \\
\hline 10. Sikingia stanleyi & 13.70 & $\mathrm{bc}$ & 17.57 & bcd & 0.17 & $\mathrm{bc}$ \\
\hline CV $(\%)$ & 9.42 & & 5.06 & & 20.73 & \\
\hline
\end{tabular}

*Tratamientos con letras desiguales difieren estadísticamente, según la prueba de Tukey $(\mathrm{P} \leq 0,05)$

Cuadro 3. Diámetro y altura total *de especies arbóreas tropicales de la segunda fase a los diez años. Quevedo, 2007.

\begin{tabular}{lcccccc}
\hline \multicolumn{1}{c}{ Especies } & Diámetro (cm) & \multicolumn{2}{c}{ Altura (m) } & \multicolumn{3}{c}{ Volumen $\left.\mathbf{( m}^{\mathbf{3}}\right)$} \\
\hline 1. Sapindus saponaria & $11.45 \mathrm{~d}^{*}$ & 8.40 & $\mathrm{~d}$ & 0.06 & $\mathrm{c}$ \\
2. Triplaris cumingiana & $15.45 \mathrm{~cd}$ & 19.19 & $\mathrm{~b}$ & 0.23 & $\mathrm{bc}$ \\
3. Cordia macrantha & $13.05 \mathrm{~d}$ & 17.03 & $\mathrm{bc}$ & 0.14 & $\mathrm{bc}$ \\
4. Artocarpus altilis & $20.85 \mathrm{bc}$ & 20.09 & $\mathrm{~b}$ & 0.44 & $\mathrm{~b}$ \\
5. Ochroma lagopus & $38,08 \mathrm{a}$ & 26.74 & $\mathrm{a}$ & 1.92 & $\mathrm{a}$ \\
6. Cordia eriostigma & $23.80 \mathrm{~b}$ & 16.68 & $\mathrm{bc}$ & 0.46 & $\mathrm{~b}$ \\
7. Cybistax donnel-smithii & $15.65 \mathrm{~cd}$ & 18.66 & $\mathrm{~b}$ & 0.23 & $\mathrm{bc}$ \\
8. Centrolobium paraense & $12.70 \mathrm{~d}$ & 16.96 & $\mathrm{bc}$ & 0.14 & $\mathrm{bc}$ \\
9. Matisia cordata & $11.95 \mathrm{~d}$ & 11.77 & $\mathrm{~cd}$ & 0.08 & $\mathrm{c}$ \\
10. Inga espectabilis & $16.45 \mathrm{~cd}$ & 20.08 & $\mathrm{~b}$ & 0.27 & $\mathrm{bc}$ \\
\hline \multicolumn{1}{c}{ CV (\%) } & $\mathbf{7 . 7 9}$ & $\mathbf{8 . 7 5}$ & & $\mathbf{2 1 . 0 2}$ & \\
\hline
\end{tabular}

*Tratamientos con letras desiguales difieren estadísticamente, según la prueba de Tukey $(\mathrm{P} \leq 0,05)$ 
Cuadro 4. Diámetro y altura total de especies arbóreas tropicales de la tercera fase a los nueve años. Quevedo, 2007.

\begin{tabular}{lccccc}
\hline \multicolumn{1}{c}{ Especies } & Diámetro (cm) & \multicolumn{2}{c}{ Altura (m) } & \multicolumn{2}{c}{ Volumen $\left.\mathbf{( m}^{\mathbf{3}}\right)$} \\
\hline 1. Cinometra bauhiniifolia & $6.45 \mathrm{~d}$ & $5.76 \mathrm{e}$ & 0.01 & $\mathrm{~d}$ \\
2. Tabebuia guayacan & $8.65 \mathrm{~cd}$ & $7.78 \mathrm{de}$ & 0.03 & $\mathrm{~d}$ \\
3. Clorophora tinctoria & $14.20 \mathrm{~b}$ & $16.70 \mathrm{a}$ & 0.17 & $\mathrm{bc}$ \\
4. Licania arborea & $11.50 \mathrm{bc}$ & $13.13 \mathrm{abc}$ & 0.09 & $\mathrm{~cd}$ \\
5. Rollinia mucosa & $12.40 \mathrm{bc}$ & $10.08 \mathrm{cde}$ & 0.08 & $\mathrm{~cd}$ \\
6. Cananga odorata & $19.05 \mathrm{a}$ & $16.24 \mathrm{cde}$ & 0.30 & $\mathrm{a}$ \\
7. Pithecellobium sp. & $13.55 \mathrm{~b}$ & $11.07 \mathrm{bcd}$ & 0.10 & $\mathrm{~cd}$ \\
8. Apeiba aspera & $18.35 \mathrm{a}$ & $14.38 \mathrm{abc}$ & 0.24 & $\mathrm{ab}$ \\
9. Cassia fistula & $11.95 \mathrm{bc}$ & $15.19 \mathrm{ab}$ & 0.11 & $\mathrm{~cd}$ \\
10. Brownea herthae & $11.70 \mathrm{bc}$ & $14.29 \mathrm{abc}$ & 0.10 & $\mathrm{~cd}$ \\
\hline CV (\%) & $\mathbf{7 . 9 8}$ & $\mathbf{9 . 8 5}$ & $\mathbf{2 4 . 0 2}$ & \\
\hline
\end{tabular}

*Tratamientos con letras desiguales difieren estadísticamente, según la prueba de Tukey $(\mathrm{P} \leq 0,05)$

Cuadro 5. Diámetro y altura total de especies arbóreas tropicales de la cuarta fase a los cinco años. Quevedo, 2007.

\begin{tabular}{|c|c|c|c|c|}
\hline \multirow{2}{*}{$\frac{\text { Especies }}{1 . \text { Nectandra spp. }}$} & \multicolumn{2}{|c|}{ Diámetro (cm) } & Altura (m) & \multirow{2}{*}{$\frac{\text { Volumen }\left(\mathbf{m}^{\mathbf{3}}\right)}{0.02 \mathrm{a}}$} \\
\hline & 7.20 & $a^{*}$ & $6.49 \mathrm{ab}$ & \\
\hline 2. Myroxilum balsamum & 9.70 & $\mathrm{a}$ & $10.03 \mathrm{ab}$ & $0.05 \mathrm{a}$ \\
\hline 3. Zanthoxylum tachuelo & 13.20 & $\mathrm{a}$ & $11.56 \mathrm{a}$ & $0.10 \mathrm{a}$ \\
\hline 4. Carapa guianensis & 7.10 & $\mathrm{a}$ & $5.66 \mathrm{ab}$ & $0.02 \mathrm{a}$ \\
\hline 5. Erythrina poeppigeana & 12.20 & $\mathrm{a}$ & $10.49 \mathrm{ab}$ & $0.08 \mathrm{a}$ \\
\hline 6. Simira ecuadorensis & 6.85 & a & $6.15 \mathrm{ab}$ & $0.02 \mathrm{a}$ \\
\hline 7. Swietenia macrophilla & 11.05 & a & $7.53 \mathrm{ab}$ & $0.07 \mathrm{a}$ \\
\hline 8. Gliricidia sepium & 11.60 & a & $10.56 \mathrm{ab}$ & $0.07 \mathrm{a}$ \\
\hline 9. Talisia setigera & 6.60 & $\mathrm{a}$ & $4.39 \mathrm{~b}$ & $0.01 \mathrm{a}$ \\
\hline 10. Erythrima smithiana & 13.60 & $\mathrm{a}$ & $7.26 \mathrm{ab}$ & $0.07 \mathrm{a}$ \\
\hline CV $(\%)$ & 29.05 & & 19.09 & 72.63 \\
\hline
\end{tabular}

*Tratamientos con letras desiguales difieren estadísticamente, según la prueba de Tukey $(\mathrm{P} \leq 0,05)$

En la cuarta fase no existe diferencias estadísticas en diámetros y volumen, pero si en altura, sobresaliendo el Z. tachuelo Little (Tachuelo) y G. sepium Jacq (Mata ratón), son árboles medianos y su crecimiento inicial es rápido (RBS, 1842; Little y Dixon, 1969); además, estas especies nativas son muy utilizadas en el medio como linderos. La especie que presentó menor diámetro, altura y volumen promedio fue T. setigera Radlk (Cebo de mico), es un árbol o arbusto delgado de 4 a 6 m de altura (Hernández y Josse, 1997) (Cuadro 5).

Incremento medio anual de las especies arbóreas tropicales de la colección de la UTEQ.
La especie que obtuvo mejor resultado en el incremento medio anual (IMA) de volumen en la primera fase de la colección, a los 11 años de edad fue $G$. arbórea (Melina) con $0.1098 \mathrm{~m}^{3}$ y la de menor IMA fue T. pentaphylla (Roble) con $0.0105 \mathrm{~m}^{3}$. En la segunda fase la especie que obtuvo mejor resultado en IMA fue O. lagopus (Balsa), quien se destaca considerablemente con $0.1960 \mathrm{~m}^{3}$ a los diez años de edad. Las especies que presentan menor IMA son $M$. cordata (Zapote)y $S$. saponaria (Jaboncillo) con $0.0087 \mathrm{~m}^{3}$ y $0.0056 \mathrm{~m}^{3}$ respectivamente (figuras 1 y 2 ). 


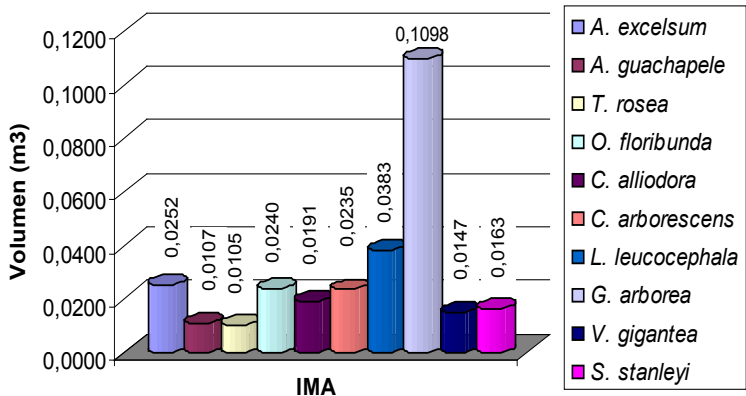

Figura 1. Incremento medio anual de los volúmenes obtenidos en la Fase I de la Colección de especies tropicales de la UTEQ. Quevedo, 2007.

La especie que obtuvo mejor resultado en el incremento medio anual (IMA) del volumen en la tercera fase de la colección a los 9 años de edad fue $C$. odorata (Cedro) con $0.0360 \mathrm{~m}^{3}$ y la de menor IMA fue C. bauhiniifolia. con $0.0015 \mathrm{~m}^{3}$. En la cuarta fase las es-

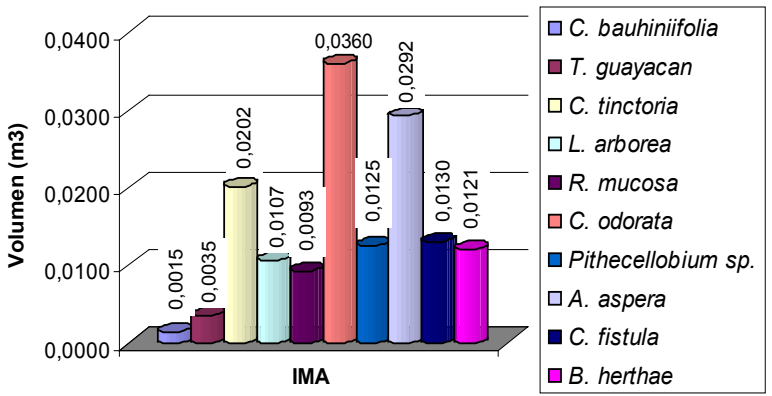

Figura 3. Incremento medio anual de los volúmenes obtenidos en la Fase III de la Colección de especies tropicales de la UTEQ. Quevedo, 2007.

Fenología de las especies arbóreas tropicales de la colección de la UTEQ.

La periodicidad de floración y fructificación de los árboles varió entre las especies observadas. Ade-

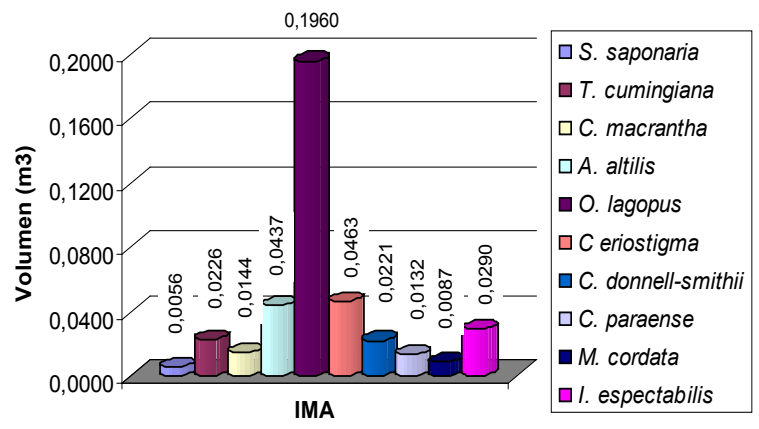

Figura 2. Incremento medio anual de los volúmenes obtenidos en la Fase II de la Colección de especies tropicales de la UTEQ. Quevedo, 2007.

pecies que reportaron mayor IMA fueron el Z. tachuelo (Tachuelo) con $0.0266 \mathrm{~m}^{3}$ y E. poepigeana (Bombón) con $0.0253 \mathrm{~m}^{3}$ a los cinco años de edad y la especie que presentó menor IMA fue T. setigera con $0.0031 \mathrm{~m}^{3}$ (figuras 3 y 4).

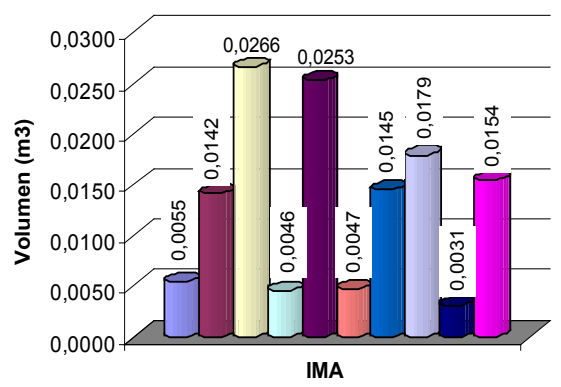

$\square$ Nectrandra sp. $\square$ M. balsamum $\square$ Z. tachuelo $\square$ C. guianensis -E. poeppigiana $\square$ S. ecuadorensis $\square$ S. macrophila $\square$ G. sepium - T. setigera $\square$ E. smithiana

Figura 4. Incremento medio anual de los volúmenes obtenidos en la Fase IV de la Colección de especies tropicales de la UTEQ. Quevedo, 2007.

más, el calendario fenológico indica que la mayoría de las especies florecen entre septiembre y marzo (Lozano, 2007). Las especies que han presentado floración y fructificación constan en el cuadro 6. 
Suatunce et al.

Cuadro 6. Floración y fructificación en la primera fase de la colección de especies arbóreas tropicales de la UTEQ. Quevedo, 2007

\begin{tabular}{|c|c|c|c|c|c|c|c|c|c|c|c|c|c|}
\hline \multirow{2}{*}{ ESPECIE } & \multirow{2}{*}{ FENOFASE* } & \multicolumn{12}{|c|}{ MES } \\
\hline & & $\mathbf{E}$ & $\mathbf{F}$ & M & $\mathbf{A}$ & $\mathbf{M}$ & $\mathbf{J}$ & $\mathbf{J}$ & $\mathbf{A}$ & $\mathbf{S}$ & $\mathbf{O}$ & $\mathbf{N}$ & D \\
\hline \multirow{2}{*}{ A. Excelsum } & Floración & $\mathrm{x}$ & & & & & & & & $\mathrm{x}$ & $\mathrm{x}$ & $\mathrm{x}$ & $\mathrm{x}$ \\
\hline & Fructificación & $\mathrm{x}$ & & & & & & & & & $\mathrm{x}$ & $\mathrm{x}$ & $\mathrm{x}$ \\
\hline \multirow{2}{*}{ O. floribunda } & Floración & & & & & & & & & $\mathrm{x}$ & $\mathrm{x}$ & $\mathrm{x}$ & \\
\hline & Fructificación & $\mathrm{x}$ & $\mathrm{x}$ & $\mathrm{x}$ & $\mathrm{x}$ & & & & & & & $\mathrm{x}$ & $\mathrm{x}$ \\
\hline \multirow{2}{*}{ C. alliodora } & Floración & $\mathrm{x}$ & $\mathrm{x}$ & $\mathrm{x}$ & & & & & & & $\mathrm{x}$ & $\mathrm{x}$ & $\mathrm{x}$ \\
\hline & Fructificación & $\mathrm{x}$ & $\mathrm{x}$ & $\mathrm{x}$ & & & & & & & & $\mathrm{x}$ & $\mathrm{x}$ \\
\hline \multirow{2}{*}{ C. arborescens } & Floración & $\mathrm{x}$ & $\mathrm{x}$ & $\mathrm{x}$ & $\mathrm{x}$ & $\mathrm{x}$ & $\mathrm{x}$ & $\mathrm{x}$ & $\mathrm{x}$ & $\mathrm{x}$ & $\mathrm{x}$ & $\mathrm{x}$ & $\mathrm{x}$ \\
\hline & Fructificación & $\mathrm{x}$ & $\mathrm{x}$ & $\mathrm{x}$ & $\mathrm{x}$ & $\mathrm{x}$ & $\mathrm{x}$ & $\mathrm{x}$ & $\mathrm{x}$ & $\mathrm{x}$ & $\mathrm{x}$ & $\mathrm{x}$ & $\mathrm{x}$ \\
\hline \multirow{2}{*}{ L. leucocephala } & Floración & $\mathrm{x}$ & $\mathrm{x}$ & & & & & & & & & $\mathrm{x}$ & $\mathrm{x}$ \\
\hline & Fructificación & $\mathrm{x}$ & $\mathrm{x}$ & $\mathrm{x}$ & $\mathrm{x}$ & $\mathrm{x}$ & $\mathrm{x}$ & $\mathrm{x}$ & $\mathrm{x}$ & $\mathrm{x}$ & $\mathrm{x}$ & $\mathrm{x}$ & $\mathrm{x}$ \\
\hline \multirow{2}{*}{ G. arborea } & Floración & $\mathrm{x}$ & & & & & & & & $\mathrm{x}$ & $\mathrm{x}$ & $\mathrm{x}$ & $\mathrm{x}$ \\
\hline & Fructificación & $\mathrm{x}$ & $\mathrm{x}$ & $\mathrm{x}$ & & & & & & & & $\mathrm{x}$ & $\mathrm{x}$ \\
\hline \multirow{2}{*}{ S. stanleyi } & Floración & & $\mathrm{x}$ & $\mathrm{x}$ & $\mathrm{x}$ & $\mathrm{x}$ & $\mathrm{x}$ & $\mathrm{x}$ & & & & & \\
\hline & Fructificación & & & $\mathrm{x}$ & $\mathrm{x}$ & & & $\mathrm{x}$ & $\mathrm{x}$ & $\mathrm{x}$ & $\mathrm{x}$ & & \\
\hline
\end{tabular}

* Fuente: Lozano 2007. Las especies que no aparecen en el cuadro se encuentran en observación y evaluación.

\section{Conclusiones}

$\mathrm{E}$ n la primera fase a pesar que las especies introducidas como Gmelina arbórea Roxb y Leucaena leucocephala Lam. muestran un crecimiento más acelerado, las especies nativas que presentaron un mayor crecimiento fueron Cordia alliodora Oken y Colubrina arborescens Sarg; mientras que en la segunda fase, además de Ochroma lagopus Sw. también sobresalieron Cordia eriostigma Pittier y Artocarpus altilis Fosberg.

En la tercera fase las especies nativas que tuvieron mayor crecimiento fueron Apeiba aspera Aubl. y Clorophora tinctoria (L.) Benth; en la cuarta fase las especies nativas con mayor crecimiento durante el primer año fueron Zanthoxylum tachuelo Little y Erythrina poeppigiana Walp. y Gliricida sepium Kunth.

Las especies que presentaron fructificación durante todo el año fueron L. leucocephala Lam. y C. arborescens Sarg.. Además, ésta ultima especie es la única que presentó floración durante todos los periodos.

\section{LiteraTURA CITADA}

Bachmann, P. 2008. Reforestación con Moral fino. Conservando el tesoro de la Naturaleza (En línea). BIOLCOM. Cumbaya- Ecuador. Consultado el 11 de marzo del 2008. Disponible en http:/www. biolcom.com $/$ moral $/$ moral.htm.

CAQ (CENTRO AGRÍCOLA DE QUITO). 1992. Manual Técnico del cultivo de la balsa. Convenio con
Corporación Andina de Fomento. Editorial Ecuador, $28 \mathrm{Pg}$.

CATIE (2004). Manual de Árboles de Centroamérica, descripciones de especies: Sapindus saponaria L; Tabebuia pentaphylla L. Pg. 865 - 868; 919 - 922. Consultado el 12 de febrero del 2008. Disponible en http://herbaria.plants.ox.ac.uk/adc/downloads/ capitulos_especies_y_anexos/ sapindus_saponaria.pdf.

Fundación Natura. 1985. Manual para la reforestación con especies exóticas y autóctonas. Quito, Ecuador. 59 p.

Hernández, C. y C. Josse. 1997. Plantas Silvestres Comestibles (en línea). Parque Nacional Machalilla, Ecuador. 78 p. Consultado el 29 de abril del 2008. Disponible en http://books.google.com.ec/ books?id=k96JWf8RW_gC\&pg=PA61\&lpg=PA6 $1 \& \mathrm{dq}=$ talisia + setigera + Radlk\&source $=$ web\&ots $=$ XbnhxBL5ir\&sig=iqLvXiwhSGvoZXK3WnqM QETZzRg\&hl=es

INEFAN. 1994. Estrategia para la industria sostenida de la madera en el Ecuador. Conocoto, Ec. 34 p.

Jadan, S., V. Valarezo. 1983. Recursos genéticos forestales en el ecuador. Qutito, Ecuador, Centro de Capacitación en Investigación forestal "Luciano Andrade Marín”. 12 p. (Informativo no. 49).

Little, E.L. \& R. G. Dixon. 1969. Árboles communes de la Provincia de Esmeraldas, Ecuador. United Nations. Development Program and Food and Agriculture Organization. Roma. 
López, M., F. Koning, H. Paredes y P. Benitez. 2002. Estimación de carbono en biomasa de bosques secundarios y plantaciones forestales en el Noroccidente del Ecuador. Proyecto CO2-GTZ, University of Gottingen, Institute of soil Science and forest nutrition. Alemania. Consultado 26 abril 2008. Disponible en http://www.gtz.de/toeb/pdf/ TOEB-estimacion-de-carbono-en-biomasa-debosques-secundarios-y-plantaciones-forestalesen-ecuador.pdf.

Louzada, J., F. De Souza. 1999. Ecología de paisagens tropicais: fracmentaçao de ecosistemas e a conservaçao de especies de Scarabaeidae. In IV Reunión Latinoamericana da Scarabaeoidología (1999, ViÇosa, MG, Brasil). 1999.

Lozano, B. 2007. Fenología de 10 especies forestales en parcelas permanentes de la UTEQ, finca Experimental "La Represa", Quevedo. Tesis de grado. UTEQ.

Manner, H. I. \& C. R. Elevitch. 2006. Species Profiles for Pacific Island Agroforestry. Cananga odorata (ylang-ylang). Consultado el 29 de abril del 2008. Disponible en http://www.agroforestry.net/tti/Cananga-ylang-ylang.pdf. www.traditionaltree.org.

Myers, N. 1984. The primary source: tropical forest and our future. W. W. Norton, New York, New York, USA.
Peacock, JM. 1995. Dryland biodiversity. In Workshop Dryland biodiversity conservation through natural resource management (1995, Amman, Jordan). 1995. Proceedings. ICARDA, Aleppo (Siria). Amman, Jordan. 64 p.

Roncacio, D. 2001. Guías técnicas para el establecimiento y manejo de plantaciones forestales productivas en el litoral ecuatoriano. Proyecto piloto para la reforestación. CORMADERA, OIMT. Quito - Ecuador.

RBS (Repertorium Botanices Systematicae). 1842. Gliricidia sepium (Jacq.) Kunth ex Walp. 1(4): 679. 1842. (En línea). Mexico, Pg. 115 - 120. Consultado el 29 de abril del 2008. Disponible en http:// www.conabio.gob.mx/conocimiento/info_especies/arboles/doctos/29-legum19m.pdf.

Zamora, N., Q. Jiménez, \& L. J. Poveda. 2000. Árboles de Costa Rical Vol II. (En línea). Centro Científico Tropical, Conservación Internacional \& Instituto Nacional de Biodiversidad. Ed. INBio. 374 p. Consultado el 29 de abril del 2008. Disponible en http://darnis.inbio.ac.cr/FMPro?-DB=UBIpub. fp3\&-lay=WebAll\&-Format=/ubi/detail.html\&$\mathrm{Op}=\mathrm{bw} \& \mathrm{id}=6397 \&-$ Find. 